\author{
В. М. Карпенко, Линь Кунь \\ Белорусский государственный университет
}

\title{
ЭКОНОМИЧЕСКОЕ РАЗВИТИЕ РЕГИОНА: ТЕОРЕТИЧЕСКИЙ АСПЕКТ
}

В статье представлен обзор существующих теорий регионального экономического развития. Произведена систематизация разнообразных методологических подходов, выполнена их сравнительная характеристика. Для каждого подхода и методологического направления выявлены их достоинства и недостатки. Авторами обоснована необходимость формирования единого методологического подхода к управлению экономическим развитием региона. В статье исследованы различные показатели, с помощью которых может быть произведена оценка качества регионального управления. Выполнена сравнительная оценка данных показателей на предмет возможности их использования в практической деятельности региональных органов управления. Все оценки сделаны с позиции возможности применения результатов исследования в управлении провинциями Китайской Народной Республики. Результаты исследований могут быть использованы в региональном управлении для устранения диспропорций в развитии регионов.

Ключевые слова: региональная экономика, наделенность факторами, региональное экономическое развитие, национальный индекс счастья, сбалансированный рост, пространственное развитие, региональная политика.

Для цитирования: Карпенко В. М., Линь Кунь. Экономическое развитие региона: теоретический аспект // Труды БГТУ. Сер. 5, Экономика и управление. 2021. № 2 (250). С. 58-68.

\author{
V. M. Karpenko, Lin Kun \\ Belarusian State University
}

\section{ECONOMIC DEVELOPMENT OF THE REGION: THEORETICAL ASPECT}

The article presents a review of existing theories of regional economic development. It systematizes the various methodological approaches, and performs their comparative characteristics. For each approach and methodological direction, their advantages and disadvantages are identified. The authors justify the necessity of forming a unified methodological approach to managing the economic development of the region. The authors studied various indicators, with the help of which the assessment of the quality of regional management can be made. A comparative assessment of these indicators for the possibility of their use in the practical work of regional governments was carried out. All assessments are made from the perspective of the possibility of applying the results of the study in the management of the provinces of the People's Republic of China. The results of the research can be used in regional management to eliminate disparities in the development of regions.

Key words: regional economy, factor endowment, regional economic development, national happiness index, balanced growth, spatial development, regional policy.

For citation: Karpenko V. M., Lin Kun. Economic development of the region: theoretical aspect. Proceedings of BSTU, issue 5, Economics and Management, 2021, no. 2 (250), pp. 58-68 (In Russian).

Введение. Вопросам регионального развития в государственной политике Китайской Народной Республики всегда отводилось важное место. Направления региональной политики сконцентрированы на повышении привлекательности территорий для жизни, работы и бизнеса; на выравнивание экономического развития провинций.

Для реализации данной цели развития необходимо теоретически обосновать, какая из теорий регионального развития может быть использована для методологической базы построения системы регионального управления.

Основная часть. На региональную экономическую теорию разделения труда наибольшее влияние оказали теория сравнительных преимуществ Д. Рикардо (1817) [1] и теория сравнительной обеспеченности (наделенности) факторами производства Э. Хекшера и Б. Олина (1933) [2].

Теория сравнительных преимуществ расширяет теорию абсолютной себестоимости, принимая в качестве основы для сравнения различия в эффективности производства отдельных факторов производства, характерные для каждой страны, а не различия в производственных издержках в качестве основы для обмена. Теория утверждает, что межрегиональная торговля и разделение труда основаны на относительных различиях в технологии производства и вытекающих из этого относительных различиях 
в себестоимости. Каждая страна или регион должны производить и экспортировать продукцию, в которой они имеют свои «сравнительные преимущества», и импортировать продукцию, в которой они имеют свои «сравнительные недостатки», в соответствии с принципом «из двух выгод выбирают большее, из двух зол выбирают меньшее».

Теория сравнительной обеспеченности (наделенности) факторами не изучает региональное разделение труда с точки зрения сравнительной разницы в себестоимостях как теория сравнительных преимуществ, а рассматривает региональное разделение труда с точки зрения разницы в эффективности производства, является развитием теории сравнительных преимуществ. Теория утверждает, что разделение труда повышает эффективность производства и общественное богатство; разница в эффективности производства является основой для регионального экономического разделения труда, а разница в производительности определяется различиями в количестве первичных факторов, которыми обладают страны; все страны должны производить те товары, которые интенсивно используют их более богатые факторы производства в обмен на импортные товары, требующие интенсивного использования их дефицитных факторов производства.

Теория сравнительных преимуществ и теория сравнительной обеспеченности (наделенности) факторами разъясняют необходимость и основные принципы разделения труда в региональной экономике, подтверждают, что региональная экономика может добиться наибольшего общественного благосостояния и эффективности использования ресурсов с помощью разделения труда. Различные регионы могут получать сравнительные преимущества за счет использования преимущественных факторов и разделения труда, что лежит в основе регионального разделения труда, региональной экономической интеграции и разработки региональной промышленной политики.

Теории сравнительных преимуществ и теории сравнительной обеспеченности (наделенности) факторами имеют общие недостатки. Во-первых, теории основаны на предпосылке, что условия производства и предложения неизменны в различных странах, факторы производства не могут свободно перемещаться между странами и отсутствует технический прогресс, т. е. отправной точкой вопроса исследования является то, что мир статичен и не интегрирован.

Во-вторых, статическое разделение труда оставляет без внимания тот факт, что технические факторы могут создавать региональные сравнительные преимущества, а также игнорирует тот факт, что по мере изменения экономического развития и времени регионы с поздним развитием имеют потенциальные преимущества (преимущества позднего развития).

В-третьих, разделение труда в соответствии с установленными сравнительными преимуществами и наделенностью факторами производства неизбежно приведет к тому, что отсталые регионы всегда будут производить сырье и первичную продукцию с низкой добавленной стоимостью, а развитые регионы всегда будут производить продукцию с высокой добавленной стоимостью, и огромная разница в добавленной стоимости продукции позволяет развитым регионам получать более богатые сравнительные преимущества. Теория сравнительных преимуществ дает повод развитым регионам захватывать дешевые ресурсы других регионов и добиваться высоких прибылей от продукции. Реализация теории в конечном итоге приведет к результату справедливого разделения труда по форме, но несправедливого распределения доходов по существу.

В-четвертых, теория не объясняет механизм распределения прибыли от разделения труда между двумя странами, т. е. как определяется цена товарообмена.

Теория пространственного развития региональной экономики в основном изучает пространственное размещение факторов производства и системы городов (поселков). К наиболее значимым теориям этой группы относятся: теория центрального места немецкого географа В. Кристаллера (1933) [3], теория сбалансированного роста британского экономиста П. Розенштейн-Родана (1943) [4], теория полюсов роста французского экономиста Ф. Перру [5], теория городских агломераций французского географа Ж. Готтмана (1957) [6], теория несбалансированного развития американского экономиста А. Хиршмана (1958) [7], теория переноса градиента американских экономистов Р. Крумма и Р. Хатора (1975) [8], а также соответствующие взгляды отечественных и зарубежных ученых.

Ключевые взгляды теории цеентрального места:

1. Как центр района, города и поселки предоставляют товары или услуги для внутренних районов или рыночных районов региона, основная функция городов - сервисный центр во внутренних районах.

2. Уровень и количество центральных мест устанавливают по определенному правилу, образуя иерархическую систему центрального места. Чем выше уровень центрального места, тем более полный и высокий ассортимент товаров и услуг он предоставляет; чем ниже уровень центра, тем меньше расстояние между ним 
и соседним центром того же уровня, а расстояние между центрами одного уровня одинаково.

3. Правильный шестиугольный рыночный район центрального места является наиболее эффективной комбинацией рыночного района для максимизации прибыли центрального места.

4. Существуют три формы иерархии центрального места, состоящего из наиболее эффективного рыночного района: система, основанная на рыночных принципах $(K=3)$; система, основанная на транспортных принципах $(K=4)$; система, основанная на административных принципах $(K=7)$. Здесь $K$ - это отношение числа центральных мест низшего уровня к числу центральных мест высшего уровня.

5. Развитие центрального места зависит от специализации его функций, а темпы развития центрального места зависят от темпов роста спроса внутренних районов на товары и услуги, предоставляемые центральным местом.

Теория центрального места разъясняет количество, масштаб и закон пространственного размещения городов в региональной городской системе, основная идея рационального планирования и разделения социально-экономических функций и общественных услуг городов на всех уровнях с помощью математических моделей внесла большой вклад в изучение экономической географии, экономики городов и теории размещения, а также стала одной из важных теоретических основ для изучения региональных экономических проблем. Однако основные предположения теории о том, что регион представляет собой «однородную территорию с однородным распределением населения с одинаковыми доходами, спросом и структурой потребления жителей», сильно отличаются от реальности и не указывают на то, насколько верно выбрано расстояние между центрами, поэтому ее практическая ценность гораздо ниже теоретической.

Ключевые взгляды теории сбалансированного роста:

1. В отсталых районах существует равновесие низшего уровня между производством и потреблением.

2. Инвестиции имеют привнесенность, и экономический рост в отсталых районах должен нарушить равновесие низкого уровня производства и потребления и сбалансированно распределить производительность по отраслям и районам, чтобы региональная экономика могла достичь сбалансированного развития.

Теория сбалансированного роста основана на неоклассической модели экономического роста с четким намерением достичь скоординированного промышленного и регионального экономического развития посредством эффекта инвестиционного мультипликатора и предотвращения попадания отстающих регионов в «замкнутый круг бедности», а ее ведущая идеология включает в себя концепцию управления системой. Но тот факт, что во времена отсталости региональной экономики в целом слаборазвитый район сам по себе не располагает ресурсами и не может добиться хороших результатов, если вкладывает ограниченный капитал в различные сектора и регионы, и региональный экономический рост может легко попасть в «ловушку равновесия низкого уровня». Даже в развитых странах или регионах такое поведение не способствует развитию сравнительных преимуществ. Поэтому теория сбалансированного регионального экономического роста практически не применялась на практике в какой-либо слаборазвитой стране или регионе с тех пор, как она была выдвинута.

Теория полюсов роста. Перру ввел «теорию ядра и периферии» Рауля Пребиша (1949) в изучение региональных экономических проблем и выдвинул концепцию полюсов регионального экономического роста. Так называемая теория полюсов роста концентрируется на одном или нескольких «центрах экономического роста» в регионе. Ключевые взгляды этой теории:

1. Сбалансированное развитие страны или региона - это лишь идеал, а региональный экономический рост в основном зависит от небольшого числа регионов и отраслей, стимулирующих развитие экономики.

2. Эти районы географически и пространственно являются городами определенного масштаба, и благодаря государственным централизованным капиталовложениям эти города развиваются, чтобы стать полюсами роста региональной экономики. Под влиянием теории полюсов роста ученые из разных стран применили ключевые взгляды в изучении региональных экономических проблем с разных точек зрения, и появились теория городских агломераций, теория несбалансированного развития, теория градиентного переноса и связанные с ними теории, образовалась относительно полная теоретическая система пространственного развития региональной экономики.

Теория городских агломераций (City group theory) считает, что городская группа состоит из центрального города и более крупных и средних городов, образующих тесные социально-экономические связи между городами посредством удобного транспорта; в процессе регионального экономического развития городская группа выполняет функции узлов транспорта и информации, инкубаторов научно-технических инноваций; городские районы и периферийные районы в городском круге достигают совместного 
использования ресурсов за счет разделения труда; городская агломерация не только создает эффективность масштабной экономики, но и способствует более быстрому развитию региональной экономики благодаря частому обмену между городами в агломерациях, высокой плотности и возникновению инновационной деятельности.

Теория несбалансированного развития (Uneven development theory) утверждает, что региональные экономические инвестиции не могут быть всеобъемлющими; региональное экономическое развитие зависит не от капиталообразования, а от эффективности использования имеющихся ресурсов; порядок приоритетности регионального промышленного развития определяется величиной «эффекта связи» отраслей; ограниченные средства должны быть объединены для поддержки более взаимосвязанных секторов и приоритетных областей.

Теория переноса градиента была разработана Круммом и Хатором путем внедрения в региональную экономику теории жизненного цикла промышленного производства профессора Гарвардского университета Раймонда Вернона (1966) и использования идей теории полюсов роста Перру и теории несбалансированного роста Хиршмана. Японский ученый К. Кодзима (1978) применил теорию к Азии и выдвинул «модель летающих гусей» градиентного переноса [9]. Основные идеи теории градиентного переноса следующие: уровень градиента регионального развития в основном определяется региональной инновационной деятельностью, которая преимущественно происходит в районах с высоким градиентом; необходимо отдавать приоритет развитию центральных городов и развитых районов с высоким градиентом для формирования «головного гуся»; с течением времени и сменой стадий жизненного цикла промышленная деятельность постепенно перемещается из районов с более высоким градиентом в районы с более низким; многоуровневая городская система является основным средством перемещения экономического градиента.

Основной взгляд теории полюсов роста и ее производной системы заключается в том, что для достижения наибольшей эффективности в использовании ресурсов инвестиции должны направляться в места с наилучшими условиями для развития, чтобы эти места могли развиваться первыми [10], а затем ждать, пока первые развитые районы возглавят развитие отсталых районов. Также считается, что «лучшие условия для развития» - это развитые регионы и крупные города, особенно центральные.

Теория полюсов роста и ее производные теории являются наиболее важными компонентами системы региональной экономической теории.
Ее основная идея состоит в приоритете эффективности и учете справедливости, что имеет большое значение для разработки стратегий регионального экономического развития в районах с относительно ограниченными ресурсами. $\mathrm{C}$ практической точки зрения эта теоретическая система более соответствует общему закону регионального экономического развития. Она хорошо адаптируется как к слаборазвитым, так и к развитым регионам и применялась в различных странах и регионах, где экономика росла эффективно и даже создавала «чудо» экономического роста. Таким образом, теория полюсов роста и ее производные теоретические системы получили широкое распространение среди ведущих региональных экономистов.

Однако недостатки теории полюсов роста и ее производных также очевидны:

1. Теория полюсов роста и ее производные теории не объясняют, следует ли ее применять в регионе с развитым рыночным механизмом или в регионе с преобладанием государственного регулирования; не указывается объективная основа для выбора региона приоритетного развития и приоритетные направления развития выбираются динамически или статически; нет объяснения того, как государство должно «действовать», когда разрыв между регионами увеличивается и разрыв в доходах достигает определенного уровня, и т. д.

2. Условия применения теории переноса градиента, вытекающей из теории полюсов роста, относительно строгие. Процесс градиентного переноса экономического развития в основном распространяется через многоуровневую городскую систему, т. е. в пределах регионального пространства, система городской сети с разумной иерархической и количественной структурой является предпосылкой для достижения градиентного переноса, иначе только разрыв нарастает. Однако пространственная иерархия и количественная структура городов в Китае, особенно в провинции Сычуань, крайне неразумны, и из-за ограничений в применении долгосрочная реализация теории градиентного переноса в Китае невозможна. Более того, теория градиентного переноса игнорирует межрегиональное экономическое развитие и интерактивность процесса модернизации, и поэтому не может быть использована в качестве теоретической основы для формулирования регионального экономического развития и модернизации в Китае [11].

3. Результаты применения теоретической системы противоречат потребностям реальности. Согласно теории полюсов роста и ее производным теориям, крупные города в развитых регионах с относительно лучшими условиями расположения 
становятся «полюсами развития», поскольку им отдается приоритет в распределении ресурсов, размещении промышленности и других преференционных политиках «удача за удачей», а их экономическая энергия в основном направляется в развитые регионы, тем самым увеличивая разрыв в региональном развитии. На самом деле, чем более отсталый регион, тем больше он нуждается в руководстве «полюса развития» для обеспечения того, чтобы деформации региональной экономической системы уменьшались, а не усугублялись. Противоречие между теоретическими результатами и реальными потребностями снижает осуществимость теории.

4. Отрицательные эффекты длительного использования значительно превысили положительные эффекты. Широко распространенное применение теории полюса роста и ее производных теорий - «модель приоритетного развития мегаполиса». Используя эту модель, экономисты и региональные руководители учитывают фокусирующий эффект мегаполиса, но в то же время они склонны переоценивать диффузионные способности экономического влияния мегаполиса и переоценивать эффект влияния развития мегаполисов на региональную экономику. На самом деле [12], влияние только одного или двух мегаполисов на региональное экономическое развитие в регионе с площадью в сотни тысяч квадратных километров очень ограничено, а «эффект обратного течения» (тенденция факторов, неблагоприятных для развития периферийных районов, перетекать в центр) центральных городов намного больше, чем их «эффект диффузии» (благотворное влияние экономического излучения центральных городов на периферию). Эффект экономического развития центральных городов почти не приносит пользы отдаленным районам, наоборот, центральные города постоянно захватывают выгодные ресурсы отдаленных районов без компенсации или по низким ценам, которые постепенно становятся придатком центральных городов. Эти районы остаются в верхней части промышленной цепи, производя сырье с низкой добавленной стоимостью и продукты первичной энергии, что приводит к «циклу накопления бедности» и оказывает большое влияние на загрязнение окружающей среды. Очевидно, что эта модель регионального экономического роста несправедлива.

Теория «преимущества отсталости» считает, что развивающиеся страны или регионы могут использовать следующие преимущества, чтобы догнать развитые страны или регионы:

1. Заимствование достижений передовой зарубежной техники и технологии для снижения затрат на исследования и разработки.
2. Обучение и подражание через накопленный мировой опыт, чтобы избежать неэффективных путей и ошибок.

3. Проведение структурных преобразований и приобретение сравнительных преимуществ в издержках, которые могут быстрее привести к конкурентоспособности.

4. Бедность побуждает к переменам и активному поиску путей развития.

Впервые теория «преимущества отсталости» разъясняет преимущества развивающейся страны или региона по сравнению с развитой страной или регионом с точки зрения теории, и эти преимущества являются движущей силой развития, позволяют им развивать экономику более эффективно и с меньшими расходами и догнать развитые страны. Теоретически она компенсировала недостатки теории регионального разделения труда, а также теории полюсов роста и ее производных теорий, которые упорно предпочитают, чтобы развитые регионы имели приоритет в развитии. Возрождение экономики «четырех маленьких драконов» в Азии и Китае изменило глобальную структуру экономического разделения труда и состав основных экономик мира, что доказало правильность теории на практике и обеспечило развивающимся регионам теоретическое обоснование для формулирования стратегий скачкообразного развития.

Недостатки теории в том, что она не является полной и строгой теоретической системой, она не разъясняет механизм образования «преимущества отсталости», не объясняет условия, при которых они могут иметь «преимущество отсталости». Более того, область исследований теории «преимущества отсталости» в основном относится к национальному макроуровню, а исследований региональных «преимуществ отсталости» и стратегий их развития на мезоуровне меньше.

Tеория перевернутого $\boldsymbol{U}$. Почти во всех странах и регионах, где реализуется теория полюсов роста и ее производные теории, наряду с экономическим ростом существует дисбаланс в региональном экономическом развитии и увеличение разрыва в доходах между населением. В своей работе «Экономическое развитие и неравенство доходов» (1955) Кузнец выдвинул перевернутую U-кривую для регионального экономического развития и обосновал, что в процессе регионального экономического развития существует перевернутая U-образная зависимость изменения размеров между разрывом экономического развития и разрывом в доходах населения. Эти разрывы не требуют вмешательства государства и автоматически уравновешиваются до определенного уровня. 
Теория перевернутого U рассматривается многими учеными региональной экономики как верный способ для объяснения применения теории полюсов роста и ее производных теорий, а также стала теоретическим обоснованием для введения плана развития мегаполиса и получения краткосрочных экономических достижений в регионах. Однако эмпирические исследования некоторых ученых показали, что в случае отсутствия активного вмешательства государства эти разрывы не обязательно достигают пика, а затем приближаются к уменьшению [13]. В процессе регионального экономического развития Китая изменения трех основных разрывов не руководствуются законом перевернутого U, а демонстрируют тенденцию к постоянному расширению [14]. Очевидно, что, согласно теории перевернутого U, в последующее длительное время невозможно было смягчить поляризацию, большой разрыв между бедными и богатыми, в результате проявились три «сельские» проблемы региональной экономики Китая.

Развитие теорий регионального управления. Теория об управленческой науке имеет долгую историю и обеспечивает наиболее важную теоретическую основу для регионального экономического управления. Среди них наибольшее руководящее значение для регионального экономического управления имеют: теория научного управления Ф. Тейлора (1911), теория управления процессами А. Файоля (1916), теория системного управления К. Барнарда (1938), теория непредвиденных обстоятельств Ф. Лутанса (1976).

Тейлор считает, что с целью достижения максимальной эффективности производства следует использовать научные методы управления, такие как стандарты и системы, чтобы заменить управление опытом. Лучший метод управления - метод управления задачами [15].

С точки зрения управления процессом эффективного функционирования организации Файоль предложил, что система «организация управление» состоит из соответствующих принципов, стандартов, методов и порядков, и четко разграничил функции управления, подчеркнув, что управление - сочетание научных методов и искусства организации и координации. Он также выдвинул 14 основных принципов, которые должны соблюдать при надлежащем управлении, включая справедливость, инициативность и т. Д. [16].

Представитель теории системного управления К. Барнард считает, что любая организация представляет собой систему сотрудничества, которая работает на двух основных принципах: «эффективность» и «мощность». «Эффективность» является предпосылкой для существования организации, «мощность» - это сочетание организационных целей и личного удовлетворения [17].

Мастер менеджмента П. Ф. Друкер (1958) выдвинул знаменитую концепцию управления по целям (Management by Objective - MBO), ocновная идея которой заключается в том, что менеджмент делает акцент на практическом применении методов. Задача менеджмента - поставить четкие организационные цели и миссии. Цель и миссию организации необходимо превратить в задачи, а ключ к достижению целей зависит от управления [18].

Ф. Лутанс (1976) в своей книге «Введение в менеджмент: наука непредвиденных обстоятельств» систематически обобщил теорию управления непредвиденными обстоятельствами. Он полагал, что не существует неизменной, общеприменимой «лучшей» теории и метода, необходимо принять соответствующую организационную структуру, стиль руководства и методы управления в соответствии с конкретными условиями и изменениями во внешней среде и гибко подходить к каждой конкретной операции управления.

Несмотря на то, что система организации региональной экономики намного больше и сложнее микроэкономического объекта, основные принципы и методы, лежащие в основе процессов управления различными объектами, совпадают. Количественный подход Тейлора к научному управлению и управление по целям Друкера предлагали парадигму для изучения региональных экономических проблем и участия в региональном экономическом управлении; принципы Файоля, которым должно следовать управление, такие как справедливость и координация, дают критерий для оценки эффективности регионального экономического управления; взгляд Лутанса на управление непредвиденными обстоятельствами показывает, как должно осуществляться региональное экономическое управление. Поэтому эти теории науки управления, которые происходят от микроэкономических объектов, также имеют важное теоретическое значение для управления региональной экономикой в Китае. Однако управление региональной экономикой является отдельным научным направлением. Как конкретно применять вышеуказанные классические теории в управлении объектом региональной экономической системы, исследования в этой области находятся только в начальной стадии. Еще не сформировалась более или менее совершенная, характерная система региональной экономической теории.

Неисчерпаемые движущие силы для управления в достижении результатов - это инновации. Знаменитый американский экономист Й. Шумпетер - «отец теории инноваций» считает, 
что «основным явлением экономического развития является инновация, а инновация - это создание новой производственной функции», т. е. введение в производственную систему «новой комбинации» факторов и условий производства, которая никогда не существовала ранее. Инновации включают новое развитие, новый прорыв технологии, разработку и использование новых рынков, появление новых комбинаций ресурсов и принятие новых способов производства (новых производственных функций и способов распределения ресурсов); функция менеджеров заключается в достижении инноваций [19]. Согласно мнению Шумпетера, инновация представляет собой технологические и институциональные инновации. Таким образом, экономическое развитие можно выразить в следующей модели:

$$
G=f(I, T),
$$

где $G$ - экономическое развитие; $I$ - система; $T$ технология.

Взаимосвязь между системой и технологией такова: производительность с технологией в качестве ядра определяет систему, а система для технологии имеет обратный эффект, система может не только способствовать технологическим инновациям, но и препятствовать им [20].

С наступлением новой экономической эры объекты управления, среда управления, цели управления, организационная структура, организационное поведение становятся все более сложными. Первоначальные теории управления и методы уже не могут удовлетворить спрос на новые теории и методы управления в новую эпоху перемен [21]. Региональная экономическая система является сложной системой, и фокус регионального экономического функционирования изменился с «эффективности прежде всего» на «руководство по качеству», а также изменились объект, среда и цель регионального экономического управления, и это требует инновации системы регионального экономического управления.

Инновационная система управления региональной экономикой основана на теории систем, согласно которой система организационного управления является открытой социальной системой, состоящей из ряда подсистем, которые могут воплощать основные функции организационного управления, включает правила, подсистему целей, техническую подсистему, подсистему организационной структуры и подсистему внешних факторов [22]. Эти четыре подсистемы независимы друг от друга, взаимодействуют друг с другом и взаимосвязаны как органическое целое.

Согласно модели экономического развития теории инноваций, можно увидеть, что ядром ее теории является «экономический масштаб», и Шумпетер полагает, что только при внедрении инноваций могут быть произведены капитал, прибыль и проценты. Очевидно, данное утверждение является очень односторонним взглядом, поскольку инновации и их поддержание являются относительными и взаимодополняющими.

Оценка качества региональной экономики. Качество - это отправная точка и конечная цель любого организационного управления. Безусловно, стремление к высокому качеству региональной экономики является основным содержанием управления региональной экономикой, а оценка качества считается наиболее фундаментальной задачей для хорошего управления.

Национальный индекс счастья (NHI). В 1970-х гг. король Бутана Дж. С. Вангчук (J. S. Wangchuck) обнаружил парадокс в модели экономического роста, а именно «ВВП превыше всего»: страна богаче, доход увеличивается, а у людей отсутствует чувство счастья. И тогда он предложил модель экономического развития, ориентированную на национальный индекс счастья (NHI). NHI включает четыре аспекта: защита окружающей среды и ресурсов, справедливое и устойчивое экономическое развитие, сохранение традиционной культуры и благое правление правительства. Он утверждал, что основная цель социально-экономического развития заключается не в росте ВВП, а в увеличении национального счастья в большей степени, чем богатства.

Согласно модели экономического развития, ориентированной на NHI, Бутан, небольшая страна в Южной Азии с доходом на душу населения менее 3000 долл. США (восьмое место в мире), занимает первое место в Азии (носит название «самого близкого к раю места»), опережая основные развитые страны мира, а Китай находится лишь на 48-м месте [23]. В настоящее время многие страны и регионы начинают использовать NHI в качестве основы для оценки качества национальной или региональной экономики. Однако применение NHI для оценки региональной экономики также имеет недостатки: его содержание представляет собой концептуальное описание на уровне правил управления, а не конкретную систему количественных показателей. Поэтому NHI довольно абстрактно и сложно в использовании, и даже если национальное счастье можно получить с помощью опросов, оно является лишь ощущением и содержит множество субъективных факторов. При одних и тех же условиях данные различаются в зависимости от места и человека. Построение системы количественной оценки является одной из насущных проблем развития региональной науки [24]. Истинное отражение развития региональной 
экономики должно иметь определенные пороговые критерии, т. е. количественные показатели оценки, а количественные показатели оценки должны обеспечивать справедливость и точность [25]. Выдвинув национальный индекс счастья, китайские и зарубежные исследовательские институты и ученые получили новое понимание оценки результатов регионального экономического развития.

Индекс человеческого развития (ИЧР) (HDI). Программа развития ООН (1990) использует индекс человеческого развития (ИЧР) для измерения степени экономического развития страны или региона [26]. ИЧР состоит из трех компонентов: знания (процент грамотности взрослых и средние годы обучения в школе), ожидаемая продолжительность жизни (ожидаемая продолжительность жизни при рождении) и жизненный уровень (реальный ВВП на душу населения в долларах США по паритету покупательной способности). ИЧР отражает научно-технический потенциал, способность распространения информации и пользования законом природы через показатель знаний; степень совершенства систем социального обеспечения и охраны здоровья через показатель продолжительности жизни; уровень экономического развития и средняя состоятельность населения - через показатель жизненного уровня. В качестве «шкалы развития, выходящей за рамки стандартов учета ВВП», ИЧР [27] может отражать основной подтекст, согласно которому конечной целью экономического развития является развитие человека. ИЧР относительно прост в управлении, однако учитывает только экономические и социальные факторы и не принимает во внимание такие факторы, как окружающая среда, транспорт и координация экономического развития. Тем более, оценочные показатели малы и не отражают истинное развитие региональной экономики комплексно.

Модели оценки PSR и EEE. Для того, чтобы компенсировать тот факт, что национальный индекс счастья (NHI) не может количественно оценить качество региональных экономик и малое количество показателей индекса человеческого развития, были разработаны две модели оценки регионального развития - PSR и EEE.

Организация экономического сотрудничества и развития (ОЭСР) и Программа ООН по окружающей среде (OECD and UNEP, 1997) предложили модель PSR (Pressur-State-Response) для оценки уровня регионального развития. Данная модель включает три первичных показателя - население, потребление и выбросы производства и жизни, инвестиции в науку и технику и охрану окружающей среды; 6 вторичных показателей об уровне промышленного развития, а также 33 третичных показателя [28].
Университет Цинхуа и японский университет Кэйо (1999) совместно предложили ЕEЕ (Energy, Economy, Environment) модель для оценки уровня регионального развития. ЕЕЕ касается трех первичных показателей - энергии, экономики и окружающей среды, в том числе энергопотребления, энергоэффективности, структуры энергетики, экономического масштаба, структуры экономики, экономического роста, экономической поддержки, выбросов трех видов промышленных отходов, индекса загрязнения и индекса защиты окружающей среды, а также более 50 третичных показателей [29].

Обе модели оценки PSR и EEE могут быть использованы для оценки и анализа текущей ситуации и политики в области энергетики, экономики и окружающей среды в регионе и могут более полностью отражать уровень регионального экономического развития. Структура их системы показателей ясна, и содержание, отражаемое каждым показателем, относительно понятно, но из-за многочисленных и пересекающихся показателей на каждом уровне правила по выбору данных показателей не единообразны и имеют определенную произвольность, что может не соответствовать действительности, и трудно собирать данные. А построенная модель слишком сложна и не сильна в работоспособности и приведет к тому, что делает ее теоретическую ценность большой, а практическую ценность малой.

Другие представительные методы оценки качества региональной экономики. На основе национального индекса счастья (NHI), индекса человеческого развития (HDI), моделей PSR и EEE Ли Синь и другие предложили систему показателей национального индекса счастья, состоящую из шести вторичных показателей - экономического положения, состояния здоровья, семейного положения, профессионального положения, социального положения и состояния внешней среды, 44 третичных показателей [30]. Учитывая недостатки моделей PSR и EEE, которые влияют на применение индекса из-за чрезмерного количества показателей, некоторые исследователи сократили определенные показатели. Ван Юнцзин и Чэн Гуанбин разработали систему оценочных показателей регионального экономического научного развития, включающую три нормативных показателя, 11 вторичных нормативных показателей, 26 конкретных оценочных показателей [24]. Лю Ян предложил 20 подпунктов показателей для оценки качества регионального экономического развития по пяти аспектам: региональная экономическая структура, региональная экономическая эффективность, способность региональных экономических связей, региональное экономическое 
благосостояние и стоимость регионального экономического развития [23]. С точки зрения практического применения Ли Юнъю предложил оценить качество региональной экономики с помощью 16 показателей на четырех уровнях: темп роста, эффективность роста, экономическая структура и социальная структура - и проанализировал экономическую стабильность провинции Цзянсу и Чжэцзяна на основе эмпирических данных [31]. Ло Сюйбин провел сравнительный анализ качества экономического развития в центральном регионе на основе четырех вторичных показателей: качество производительности, экономическая структура, технологический прогресс и развитие человеческих ресурсов, а также 13 третичных показателей [32].

Заключение. Анализ теорий регионального развития и методических подходов к оценке качества региональной экономики показал, что все они в целом могут объективно оценивать региональное экономическое развитие, являются актуальными и применимыми на практике. Тем не менее в силу того, что в них уделяется чрезмерное внимание экономической эффективности, или факторы оценки трудно поддаются количественной оценке, или сбор данных по оценочным показателям затруднен, или модель оценки является слишком сложной из-за слишком большого числа показателей, их прикладная ценность недостаточна, и существует не так много систем индексов оценки качества региональной экономики, которые действительно отражают «справедливость, координацию и способность к экономическому росту». Методическое обеспечение процесса регионального управления нуждается в дальнейшем совершенствовании и развитии.

\section{Список литературы}

1. Ricardo D. Political Economy and Taxation Principles. Beijing: China Publishing House, 2015. 220 p.

2. Bertier O. Interregional and international trade. Beijing: The Commercial Press, 2016. 340 p.

3. Christaller W. Central Places in Southern Germany. Beijing: The Commercial Press, 2018. 264 p.

4. Rosenstein-Rodan Paul N. Problems of Industrialization of Eastern and Southastern Europa // Economic Journal. 1943. No. 53 (210/211). P. 202-211.

5. Perroux F. Economic Space: Theory and Application // Quarterly Journal of Economics. 1950. Vol. 64. P. 44.

6. Gottmann J. Megalopolis or the Urbanization of the Northeastern Seaboard // Economic Geography. 1957. Vol. 33, no. 3. P. 198-200.

7. Hirschman A. O. Strategy of Economic Development // Yale University Press. 1958. No. 17. P. 217.

8. Zhu Chuangeng. Regional Economics // China Social Sciences Press. 2021. No. 6. P. 136-145.

9. Xiaodaoqing. Foreign Trade Theory. Tianjin: Nankai University Press, 1987. 312 p.

10. Sun Jiuwen. An Empirical Study on the Transformation of China's Regional Economic Structure. Beijing: China Light Industry Press, 2019. 312 p.

11. Gao Hongye. Regional Economics. Beijing: Renmin University of China Press, 2020. 276 p.

12. Rao Huilin. Urban Economics. Dalian: Dongbei University of Finance and Economics Press, 2017. 670 p.

13. Wang Jiangui. Is the inverted U phenomenon an economic law? // Economic Research. 2020. No. 7. P. 63-67.

14. Li Xiumin. A comparative study of the effects of urbanization on economic growth in eastern, central and Sichuan provinces of China // Proceedings of the International Symposium on the Coordinated Urban and Rural Development and Social Innovation in Sichuan Province, China. Chongqing. 2016. No. 11. P. 97-108.

15. Taylor F. Principles of Scientific Management. Beijing: Mechanical Industry Press, 2016. 214 p.

16. Fayol A. Industrial management and general management. Beijing: Mechanical Industry Press, 2019. $276 \mathrm{p}$.

17. Barnard Ch. Organization and management. Beijing: Renmin University of China Press, 2019. 235 p.

18. Drucker F. P. Management practice. Beijing: Workers' Publishing House, 2019. 287 p.

19. Schumpeter J. Economic Development Theory. Beijing: The Commercial Press, 2020. 389 p.

20. Ma Hongwei. Economic development and institutional innovation// Economic Review. 2016. No. 1. P. 36-42.

21. Song Xuefeng. Research progress of management complexity // Science and Technology Review. 2020. No. 10. P. 55.

22. Kast Frement E., Rosenweig J. Organization and management: A system and contingency approach. New York: Mc Graw-Hill, 2015. 286 p.

23. Yin Yiwen. Revelation from Bhutan: Replace GDP with Gross National Happiness // Leadership Collection. 2019. No. 21. P. 138-142.

24. Liu Yang. The construction and evaluation of the quality index system of regional economic development under the guidance of the scientific development concept // Journal of Harbin University of Commerce (Social Science Edition). 2019. No. 1. P. 94-96. 
25. Wang Yongjing, Cheng Guangbin. The Index System of the Scientific Outlook on Development: Framework and Evaluation Methods // New Theory on Statistics. 2018. No. 3. P. 9-11.

26. United Mations Development Programme // Human Development Report. 2020. No. 10. P. 46.

27. Wang Zhiping. Human Development Index (HDI): meaning, methods and improvements // Journal of Shanghai Administration Institute. 2017. No. 3. P. 47-57.

28. UNCSD. Indicators of Sustainable Development: Guidelines and Methodologies // United Wations. 2017. No. 10. P. 43.

29. Zhang Aling, Li Jifeng. Constructing China's energy-economy-environment system evaluation model // Journal of Tsinghua University (Nan Science Edition). 2017. No. 9. P. 1537-1540.

30. Li Xin, Lai Yangen, Tan Min. Construction of National Happiness Index System // Southeast Academic. 2019. No. 5. P. 66-75.

31. Li Yongyou. An Empirical Study on the Quality of Economic Development // Finance and Trade Economics. 2018. No. 8. P. 113-118.

32. Luo Xubin. Evaluation of the quality of economic development in the central region // Contemporary Economy. 2019. No. 7. P. 82-83.

\section{References}

1. Ricardo D. Political Economy and Taxation Principles. Beijing, China Publishing House, 2015. 220 p.

2. Bertier O. Interregional and international trade. Beijing, The Commercial Press, 2016. 340 p.

3. Christaller W. Central Places in Southern Germany. Beijing, The Commercial Press, 2018. 264 p.

4. Rosenstein-Rodan Paul N. Problems of Industrialization of Eastern and Southastern Europa. Economic Journal, 1943, no. 53 (210/211), pp. 202-211.

5. Perroux F. Economic Space: Theory and Application. Quarterly Journal of Economics, 1950, vol. 64, p. 44.

6. Gottmann J. Megalopolis or the Urbanization of the Northeastern Seaboard. Economic Geography, 1957, vol. 33, no. 3, pp. 198-200.

7. Hirschman A. O. Strategy of Economic Development. Yale University Press, 1958, no. 17, p. 217.

8. Zhu Chuangeng. Regional Economics. China Social Sciences Press, 2021, no. 6, pp. 136-145.

9. Xiaodaoqing. Foreign Trade Theory. Tianjin, Nankai University Press, 1987. 312 p.

10. Sun Jiuwen. An Empirical Study on the Transformation of China's Regional Economic Structure. Beijing, China Light Industry Press, 2019. 312 p.

11. Gao Hongye. Regional Economics. Beijing, Renmin University of China Press, 2020. 276 p.

12. Rao Huilin. Urban Economics. Dalian, Dongbei University of Finance and Economics Press, 2017. $670 \mathrm{p}$.

13. Wang Jiangui. Is the inverted U phenomenon an economic law? Economic Research, 2020, no. 7, pp. 63-67.

14. Li Xiumin. A comparative study of the effects of urbanization on economic growth in eastern, central and Sichuan provinces of China. Proceedings of the International Symposium on the Coordinated Urban and Rural Development and Social Innovation in Sichuan Province, China. Chongqing, 2016, no. 11, pp. 97-108.

15. Taylor F. Principles of Scientific Management. Beijing, Mechanical Industry Press, 2016. 214 p.

16. Fayol A. Industrial management and general management. Beijing, Mechanical Industry Press, 2019. $276 \mathrm{p}$

17. Barnard Ch. Organization and management. Beijing, Renmin University of China Press, 2019. 235 p.

18. Drucker F. P. Management practice. Beijing, Workers' Publishing House, 2019. 287 p.

19. Schumpeter J. Economic Development Theory. Beijing, The Commercial Press, 2020. 389 p.

20. Ma Hongwei. Economic development and institutional innovation. Economic Review, 2016, no. 1, pp. 36-42.

21. Song Xuefeng. Research progress of management complexity. Science and Technology Review, 2020 , no. 10 , p. 55

22. Kast Frement E., Rosenweig J. Organization and management: A system and contingency approach. New York, Mc Graw-Hill, 2015. 286 p.

23. Yin Yiwen. Revelation from Bhutan: Replace GDP with Gross National Happiness. Leadership Collection, 2019, no. 21, pp. 138-142.

24. Liu Yang. The construction and evaluation of the quality index system of regional economic development under the guidance of the scientific development concept. Journal of Harbin University of Commerce (Social Science Edition), 2019, no. 1, pp. 94-96.

25. Wang Yongjing, Cheng Guangbin. The Index System of the Scientific Outlook on Development: Framework and Evaluation Methods. New Theory on Statistics, 2018, no. 3, pp. 9-11. 
26. United Mations Development Programme. Human Development Report, 2020, no. 10, p. 46.

27. Wang Zhiping. Human Development Index (HDI): meaning, methods and improvements. Journal of Shanghai Administration Institute, 2017, no. 3, pp. 47-57.

28. UNCSD. Indicators of Sustainable Development: Guidelines and Methodologies. United Wations, 2017, no. 10, p. 43.

29. Zhang Aling, Li Jifeng. Constructing China's energy-economy-environment system evaluation model. Journal of Tsinghua University (Nan Science Edition), 2017, no. 9, pp. 1537-1540.

30. Li Xin, Lai Yangen, Tan Min. Construction of National Happiness Index System. Southeast Academic, 2019, no. 5, pp. 66-75.

31. Li Yongyou. An Empirical Study on the Quality of Economic Development. Finance and Trade Economics, 2018, no. 8, pp. 113-118.

32. Luo Xubin. Evaluation of the quality of economic development in the central region. Contemporary Economy, 2019, no. 7, pp. 82-83.

\section{Информация об авторах}

Карпенко Валерий Михайлович - кандидат технических наук, доцент, доцент кафедры инноватики и предпринимательской деятельности. Белорусский государственный университет (220010, г. Минск, пр-т Независимости, 4, Республика Беларусь). E-mail: vmkarpenka@gmail.com

Линь Кунь - аспирант кафедры международного менеджмента. Белорусский государственный университет (220010, г. Минск, пр-т Независимости, 4, Республика Беларусь). E-mail: 79393047@qq.com

\section{Information about the authors}

Karpenko Valeriy Mikhaylovich - PhD (Engineering), Associate Professor, Assistant Professor, the Department of Innovation and Entrepreneurship. Belarusian State University (4, Nezavisimosti Ave., 220010, Minsk, Republic of Belarus). E-mail: vmkarpenka@gmail.com

Lin Kun - PhD student, the Department of International Management. Belarusian State University (4, Nezavisimosti Ave., 220010, Minsk, Republic of Belarus). E-mail: 79393047@qq.com 